\title{
Traffic-aware Resource allocation with aggregation in Heterogeneous Networks with WLANs
}

\author{
Haeyoung Lee, Seiamak Vahid, and Klaus Moessner \\ Institute for Communcation Systems (ICS), University of Surrey, U.K. \\ Email: \{Haeyoung.Lee, S.Vahid, K.Moessner\}@ surrey.ac.uk
}

\begin{abstract}
We consider resource allocation with aggregation for different types of traffic in heterogeneous networks, including WLANs. While mobile data traffic is expected to increase, efficient management of multiple bands including unlicensed band becomes increasingly important. In this context, we formulate a resource allocation problem using utility functions for heterogeneous traffic and propose a novel algorithm that considers the estimated UE speed, traffic types and channel quality. Simulation results illustrate performance of the proposed algorithm in terms of higher utility value and fairness, even at high traffic loads. Additional improvements in resource utilization through estimating UE speed and allocating low-mobility UEs to Wi-Fi are shown.
\end{abstract}

Index Terms-Carrier aggregation, load balancing, LWA

\section{INTRODUCTION}

Mobile data traffic is expected to increase around $45 \%$ annually, resulting in a ten-fold increase in total by 2021 compared to 2015 . Almost $70 \%$ of the traffic will be video, which indicates $55 \%$ of the annual growth [1]. To meet this burgeoning demand, the use of multiple spectrum bands has been considered. In 3GPP, carrier aggregation (CA) has been standardized and Rel-13 targets aggregation of up to 32 component carriers (CCs). Along with the licensed band, the operators are turning to use unlicensed bands to offload their cellular traffic, and several technologies have been investigated, i.e., LTE for Unlicensed (LTE-U) or Licensed Assisted Access (LAA)-LTE [2] and LTE-WLAN Aggregation (LWA) [3]. Both LTE-U and LAA require new 5 GHz LTEenabled devices for unlicensed band use whilst LWA uses unlicensed band through $\mathrm{Wi}-\mathrm{Fi}$ and does not need any new device hardware, just Wi-Fi access points (APs) connected to LWA base stations (BSs) [4]. Unlicensed bands are becoming increasingly important to meet growing traffic demand while licensed bands remain critical to deliver advanced services. For efficient use of multiple spectrum bands with different regimes, the resource allocation for multiple bands becomes a necessity to support the quality of service (QoS) of heterogeneous traffic types [5].

In literature, there have been efforts on developing mechanisms to support QoS of heterogeneous traffic types, and the utility function approach has been investigated widely [6]. In [7], rate allocation using multiple carriers is studied to support different traffic types with utility functions. Utilization of unlicensed bands is added in [8]. In [9] [10], traffic load balancing in multiple Radio Access Technologies (RATs) has been investigated with utility functions. While aforementioned works were conducted to support QoS of different traffic types or offload cellular traffic, to the best of our knowledge, the issue of how to efficiently utilize multiple bands from heterogeneous networks, including WLANs for heterogeneous traffic UEs capable of aggregation, has not been fully scrutinized.

In this paper, we consider the problem of resource allocation with aggregation of multiple bands including unlicensed bands (via $\mathrm{Wi}-\mathrm{Fi}$ ) for support of various traffic types. In this contribution, allocation of primary and secondary carriers is carried out as a two-step process, and the optimal primary carriers are allocated from licensed bands by applying the Hungarian method, similar to the approach in our previous work [11]. For UEs requiring higher data rates, supplemental carriers are allocated either from licensed bands or with Wi-Fi links. By calculating an access index integrating the estimated UE's speed, traffic types and channel quality, the proposed approach selects the most suitable UEs to access Wi-Fi link. For UEs not selected for use of Wi-Fi links, secondary carriers are allocated in licensed bands considering utility functions. While the proposed approach prioritizes UEs of inelastic traffic over UEs with elastic traffics in resource allocation, it prefers UEs with low mobility, stronger Wi-Fi signal and elastic traffic to select Wi-Fi links. From the simulation study, the proposed approach is shown to improve QoS provided to UEs by $39.45 \%$ in terms of the utility value, and the fairness of UE's utility values by $52 \%$ even when UEs move fast.

The organization of the paper is as follows. Section II describes the system model including the utility functions of different traffic types. In Section III, the resource allocation problem is formulated. Then, the proposed algorithm is presented in Section IV and its performance is evaluated in Section V. This paper is concluded in Section VI.

\section{System AND Channel Model}

\section{A. System Model}

We consider the downlink transmission of the OFDM based cellular network coexisting with WLAN users, as depicted in Fig. 1. The system consists of a cellular BS, a set of users (UEs) $\mathcal{K}$, having cellular (e.g., LTE) and Wi-Fi air interfaces. There are also a $\mathrm{Wi}-\mathrm{Fi} \mathrm{AP}$ and $W$ wireless nodes (WNs) with only Wi-Fi air interface. It is assumed that BS and AP can communicate based on the Xw interface [12]. The mobile system has its dedicated licensed spectrum over multiple bands, with a set of carriers $\mathcal{N}$. Multiple carriers can be integrated for transmission using carrier aggregation, and 


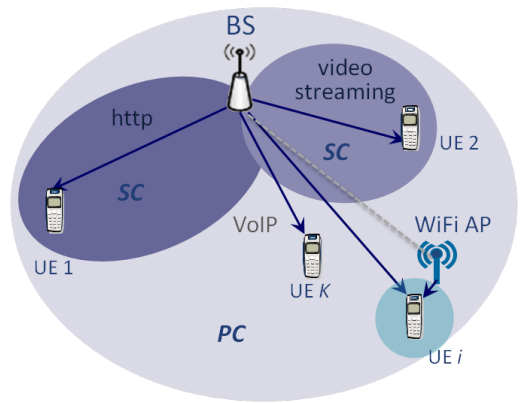

Fig. 1. Downlink transmission with carrier aggregation to multiple users having different traffic types in the heterogeneous networks including WLANs

the aggregated channel consists a primary carrier (PC) and multiple secondary carriers (SCs). While the PC provides a reliable connection, SCs can provide higher rates and more capacity [13]. In addition, the Wi-Fi links as SCs can be aggregated to improve data rates, similarly to the LWA scheme [12].

The traffic is classified into elastic traffic from applications including FTP and HTTP, and inelastic traffic from real-time applications such as VoIP, video streaming and etc. [11].

\section{B. Channel Model}

Carrier $j$ between the BS and UE $i$ is assumed to undergo independent and identically distributed (i.i.d.) Rayleigh fading. The channel coefficient $h_{i j}$, is a complex Gaussian random variable with zero mean and variance $\sigma_{h_{i j}}^{2}$, i.e., $h_{i j} \sim \mathcal{C N}\left(0, \sigma_{h_{i j}}^{2}\right)$. Suppose that the channel state information (CSI) is perfectly known at the receiver. For the transmission from $\mathrm{BS}$ to $\mathrm{UE} i$ with carrier $j$, the received signal to noise ratio (SNR) can be expressed as

$$
\rho_{i j}=\frac{\left|h_{i j}\right|^{2} P_{i j}}{\sigma^{2}}=\frac{g_{i j} P_{i j}}{\sigma^{2}},
$$

where $g_{i j}=\left|h_{i j}\right|^{2}$ of a Chi-square distributed random variable for all $i, j . P_{i j}=P_{\max } / N$ is the transmit power for equal power allocation for a given $P_{\max }$ and $\sigma^{2}$ is the variance of additive white Gaussian noise (AWGN). The data rate is

$$
r_{i j}=W_{j} \cdot \log _{2}\left(1+\rho_{i j}\right)
$$

where $W_{j}$ is the bandwidth of carrier $j$. Consider the BS can use multiple carriers of licensed bands and the Wi-Fi link by aggregation for transmission. The overall data rate to $\mathrm{UE} i, r_{i}$ is given by

$$
r_{i}=\sum_{j \in \mathcal{N}} \alpha_{i j} \cdot r_{i j}+\alpha_{i}{ }^{w} \cdot r_{i}{ }^{w},
$$

where $\alpha_{i j}$ is the carrier allocation indicator, i.e., $\alpha_{i j}=1$ indicates that carrier $j$ is allocated to UE $i$ and $\alpha_{i j}=0$, otherwise. $\alpha_{i}{ }^{w}$ is the Wi-Fi link allocation indicator and the achievable data rate from the Wi-Fi link $r_{i}{ }^{w}$ is defined as

$$
r_{i}{ }^{w}=t_{i}{ }^{w} \times W^{w} \log _{2}\left(1+\rho_{i}{ }^{w}\right),
$$

where $W^{w}$ is the Wi-Fi channel bandwidth and $\rho_{i}{ }^{w}$ is the SNR of the Wi-Fi channel and calculated similarly in (1). $t_{i}{ }^{w}$ is the time fraction for the Wi-Fi link utilized to transmit the data to UE $i$ and calculated with the successful transmission probability over Wi-Fi given by [14]

$$
t_{i}{ }^{w}=\tau(1-\tau)^{W+N_{W}-1}
$$

where $W$ is the number of Wi-Fi devices and $N_{W}$ is the number of UEs utilizing the Wi-Fi link, i.e., $N_{W}=\sum_{i} \alpha_{i}{ }^{w}$. $\tau$ is the stationary probability that Wi-Fi users transmit a packet and can be written as [15]

$$
\tau=\frac{2\left(1-2 \hat{P}_{c}\right)}{\left(1-2 \hat{P}_{c}\right)\left(B_{m}+1\right)+\hat{P}_{c} B_{m}\left[1-\left(2 \hat{P}_{c}\right)^{C_{s}}\right]},
$$

where $B_{m}$ is the minimum backoff window size, $C_{s}$ is the maximum contention stage, and $\hat{P}_{c}$, the collision probability is defined by [15]

$$
\hat{P}_{c}=1-(1-\tau)^{W+N_{W}-1} .
$$

Given the channel quality and the traffic load of the Wi-Fi, the scheduler at BS allocates resources in licensed bands and Wi-Fi links.

\section{Applications Utility Functions}

To allocate resources considering the characteristics of different traffic, application utility functions are considered. The function of UE $i, U_{i}\left(r_{i}\right)$ is represented by a sigmoidallike function or a logarithmic function [11]. The normalized logarithmic utility function for elastic traffic UEs can be represented as

$$
U_{i}\left(r_{i}\right)=\frac{\log \left(1+k_{i} r_{i}\right)}{\log \left(1+k_{i} r_{i}^{\max }\right)},
$$

where $r_{i}^{\max }$ is the required rate for UE $i$ to achieve $100 \%$ utilization and $k_{i}$ is the increasing rate of utility percentage with the allocated rate $r_{i}$. For inelastic traffic UEs, the normalized sigmoidal-like utility function is utilized as follows.

$$
U_{i}\left(r_{i}\right)=c_{i}\left(\frac{1}{1+e^{-a_{i}\left(r_{i}-b_{i}\right)}}-d_{i}\right),
$$

where $c_{i}=\left(1+e^{a_{i} b_{i}}\right) / e^{a_{i} b_{i}}$ and $d_{i}=1 /\left(1+e^{a_{i} b_{i}}\right)$. The utility parameters $k_{i}, a_{i}$ and $b_{i}$ can be set depending on QoS of traffic types. Considering (3), $U_{i}\left(r_{i}\right)$ in (8) and (9) can be represented as a multi-variable function.

\section{PROBLEM FORMULATION}

To allocate resources of carriers in a licensed band and the Wi-Fi link to multiple UEs, we consider the utility proportional fairness (PF) objective function formulation in (10).

$$
\begin{aligned}
P: \max _{\alpha_{i j}, \alpha_{i}^{w}} & \prod_{i \in \mathcal{K}} U_{i}\left(\sum_{j \in \mathcal{N}} \alpha_{i j} r_{i j}+\alpha_{i}^{w} r_{i}^{w}\right), \\
\text { s.t. } & \sum_{i \in \mathcal{K}} \alpha_{i j} \leq 1, \forall j \in \mathcal{N}, \\
& \alpha_{i j}, \alpha_{i}^{w}=\{0,1\}, \forall i \in \mathcal{K}, \forall j \in \mathcal{N}, \\
& \sum_{j \in \mathcal{N}} \alpha_{i j} r_{i j}+\alpha_{i}^{w} r_{i}^{w} \geq r_{i}^{r e q}, \forall i \in \mathcal{K} .
\end{aligned}
$$


The objective of problem $P$ in (10) is to allocate resources to maximize the total system utility while ensuring proportional fairness between UEs (i.e., the product of the utilities of UEs). It considers allocation of carriers in licensed bands with $\alpha_{i j}$ as well as utilization of the Wi-Fi link with $\alpha_{i}^{w}$. While $\alpha_{i j}$ is the allocation indicator as shown in (12), each carrier is exclusively allocated to one user, thus the constraint (11) is imposed. For UE $i$, the rate requirement $r_{i}^{r e q}$ is considered to guarantee the QoS in (13).

\section{RESOURCE ALLOCATION AND AGGREGATION}

One of the challenging aspects to the solution of $P$ in (10) is the discrete nature of carrier assignment, and when coupled with QoS constraints, makes the problem even harder to solve. Additionally, while the data rate from the Wi-Fi link, $r_{i}^{w}$ is not fixed, but varies depending on how many UEs are finally selected for utilizing the Wi-Fi link (i.e., $\sum_{i} \alpha_{i}^{w}$ ) as shown in (4) and (5), the problem becomes more difficult. Moreover, under practical scenarios in LTE-Advanced, when a UE (re)establishes a radio resource control (RRC) connection with the BS, the primary component carrier (PCC) is configured (for initial control and data traffic). Then, depending on traffic load and QoS requirements, one or more secondary component carriers (SCCs) are configured to increase the data rate [11]. The Wi-Fi links can also be an option for use as supplemental carriers. Thus, the PCC should be robust, and is typically chosen such that it provides the most ubiquitous coverage and/or best signal quality [13]. For selection of SCCs, the different characteristics of licensed and unlicensed bands should be considered as well as the different channel quality. Considering this, we propose a novel resource allocation and aggregation algorithm.

Fig. 2 illustrates the flowchart of the proposed approach. Firstly, for given carriers in licensed bands, a single optimal primary carrier is determined for each UE. For UEs requiring higher data rates, there are two options for secondary carrier allocation: 1) using a Wi-Fi link or 2) allocating carriers from licensed bands. While exploitation of Wi-Fi links can increase the data rate without the use of licensed band, the gain would not be promising at very high traffic loads in the Wi-Fi networks. When the traffic load in Wi-Fi network is not

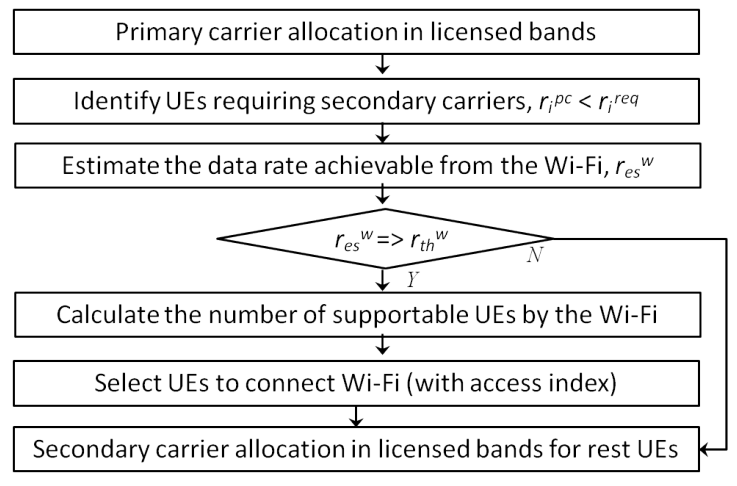

Fig. 2. Flowchart of the proposed approach high, (i.e., the estimated rate achievable from Wi-Fi $r_{e s}^{w}$ is not smaller than its threshold $r_{t h}^{w}$ ), Wi-Fi access will be considered and UEs for $\mathrm{Wi}-\mathrm{Fi}$ will be decided. The access index indicating UE suitability to access Wi-Fi is calculated based on UE's estimated mobility, the received power of the Wi-Fi signal, and the traffic types. Then UEs having higher index values will be chosen for using the Wi-Fi link. For UEs not chosen for Wi-Fi, secondary carrier allocation is carried out using the licensed bands. We present the details of each step for the proposed resource allocation and aggregation algorithm in the following subsections.

\section{A. Resource allocation for Primary Carriers}

While the primary carrier is to set up a robust connection, we select a group of carriers $\mathcal{G}_{p}$ consisting of $K$ of carriers, with good channel characteristics from the lower licensed band. We can denote $\mathcal{G}_{s}$ for carriers not included in $\mathcal{G}_{p}$. The primary carrier allocation problem is formulated as follows.

$$
\begin{array}{ll}
P 1: & \max _{\alpha_{i j}} \prod_{i \in \mathcal{K}} U_{i}\left(\sum_{j \in \mathcal{G}_{p}} \alpha_{i j} r_{i j}\right) \\
\text { s.t. } & \sum_{i} \alpha_{i j}=1, \forall j \in \mathcal{G}_{p}, \sum_{j} \alpha_{i j}=1, \forall i \in \mathcal{K}, \\
& \alpha_{i j} \in\{0,1\}, \forall i \in \mathcal{K}, j \in \mathcal{G}_{p} .
\end{array}
$$

Note $\arg \max \prod U_{i}(\cdot)$ is equivalent to $\arg \max \sum \log \left(U_{i}(\cdot)\right)$. Since $P 1$ becomes the one-to-one matching problem (between 'UE' and 'carrier'), we apply the Hungarian algorithm, a well-known approach to solve one-to-one matching problem in polynomial time optimally. From the allocated primary carriers, the data rate $r_{i}^{p c}=\sum_{j} \alpha_{i j} r_{i j}$ could be achievable. For UEs requiring higher data rates (i.e., $r_{i}^{p c}<r_{i}^{r e q}$ ), secondary carriers allocation can be carried out either from resources in the licensed band or by using a Wi-Fi link.

\section{B. Exploitation of the Wi-Fi link}

To find proper UEs for the Wi-Fi link, the access index of UEs consisting of mobility index, Wi-Fi signal strength index [16] and traffic index is calculated. Considering the characteristics of Wi-Fi networks, we select UEs of less mobility, stronger Wi-Fi signal and elastic traffic.

Firstly, the average received power of Wi-Fi signal from UE $i, \bar{P}_{i}^{w}$ and its standard deviation, $\sigma_{i}^{p}$ are calculated as follows.

$$
\bar{P}_{i}^{w}=\frac{1}{T} \sum_{t=0}^{T-1} P_{i}^{w}(t), \quad \sigma_{i}^{p}=\sqrt{\frac{1}{T} \sum_{t=0}^{T-1}\left(P_{i}^{w}(t)-\bar{P}_{i}^{w}\right)^{2}},
$$

where $t$ denotes the time slot, $P_{i}^{w}(t)$ is the received power at $t, \bar{P}_{i}^{w}$ denotes the average in the latest $T$ slots. By using (17), the mobility index can be calculated as,

$$
I_{i}^{M}(t)=e^{-\sigma_{i}^{p}}
$$

While $I_{i}^{M}(t) \in(0,1], I_{i}^{M}(t)$ close to 1 indicates low mobility.

The signal strength index can also be defined by the received signal power and its max value as follows.

$$
I_{i}^{S}(t)=P_{i}^{w}(t) / P_{\max }^{w} .
$$


The traffic index considering the traffic type of UEs is defined as,

$$
I_{i}^{T}(t)= \begin{cases}\frac{\# \text { of UEs of elastic traffic }}{\text { \# of total UEs }}, & \text { for elastic traffic UE } \\ 0, & \text { otherwise. }\end{cases}
$$

The access index for UE $i$ is defined as a sum of weighted three indices as follows.

$$
I_{i}^{A}(t)=\lambda_{1} I_{i}^{M}(t)+\lambda_{2} I_{i}^{S}(t)+\lambda_{3} I_{i}^{T}(t),
$$

where $\lambda_{1}, \lambda_{2}$ and $\lambda_{3}$ are weight factors of each index. While each factor has the range of $[0,1]$, their sum becomes 1 . If the value of $\lambda_{1}, \lambda_{2}$ or $\lambda_{3}$ is closer to 1 , the allocation gives higher priority to mobility, the signal strength, or traffic type, respectively. A higher access index of the UE means it is more beneficial to use the Wi-Fi link, as it moves less, receives a stronger signal and supports elastic traffic.

\section{Resource allocation for Secondary Carriers}

While use of a Wi-Fi link can increase the data rate of UEs, access of many devices decreases the time fraction for the $\mathrm{Wi}$ Fi link occupied by the BS to transmit the data to each device as shown in (5). Thus, after some UEs are chosen for the use of Wi-Fi link, the secondary carriers for rest UEs $\mathcal{K}_{s}$ can be allocated from the licensed band, $\mathcal{G}_{s}$. The secondary carrier allocation can be formulated as follows.

$$
\begin{aligned}
P 2: \max _{\alpha_{i j}} & \prod_{i \in \mathcal{K}_{s}} U_{i}\left(r_{i}^{p c}+\sum_{j \in \mathcal{G}_{s}} \alpha_{i j} r_{i j}\right), \\
\text { s.t. } & \sum_{i \in \mathcal{K}_{s}} \alpha_{i j} \leq 1, \forall j \in \mathcal{G}_{s}, \\
& \alpha_{i j}=\{0,1\}, \forall i \in \mathcal{K}_{s}, \forall j \in \mathcal{G}_{s}, \\
& r_{i}^{p c}+\sum_{j \in \mathcal{G}_{s}} \alpha_{i j} r_{i j} \geq r_{r e q}^{i}, \forall i \in \mathcal{K}_{s} .
\end{aligned}
$$

The problem $P 2$ is a combinatorial one due to the binary variable $\alpha_{i j}$, which makes the problem intractable for large system. To simplify the problem, $\alpha_{i j}$ is relaxed to continuous variables to take any real value in range of $[0,1]$. Then, the problem becomes convex with concave feasibility region. We analyze the simplified problem using the Lagrange multiplier.

Let $\mathcal{L}(\boldsymbol{\alpha}, \boldsymbol{\gamma}, \boldsymbol{\lambda}, \boldsymbol{\mu})$ be the Lagrangian given by

$$
\begin{array}{r}
\mathcal{L}(\boldsymbol{\alpha}, \boldsymbol{\gamma}, \boldsymbol{\lambda}, \boldsymbol{\mu})=\sum_{i} \log \left(U_{i}\left(r_{i}^{p c}+\sum_{j} \alpha_{i j} r_{i j}\right)\right)+\sum_{i} \sum_{j} \gamma_{i j} \alpha_{i j} \\
-\sum_{j} \lambda_{j}\left(\sum_{i} \alpha_{i j}-1\right)+\sum_{i} \mu_{i}\left(r_{i}^{p c}+\sum_{j} \alpha_{i j} r_{i j}-r_{r e q}^{i}\right),
\end{array}
$$

where $\gamma_{i j}, \lambda_{j}$, and $\mu_{i}$ are non-negative Lagrangian multipliers. Let us differentiate $\mathcal{L}$ with respect to $\alpha_{i j}$ and apply the Karush-Kuhn-Tucker (KKT) conditions which are necessary and sufficient for the optimality of a given set of constraints.

$$
\begin{gathered}
\frac{\partial \mathcal{L}}{\partial \alpha_{i j}}=\left[\bar{U}_{i}^{\prime}\left(r_{i}\right)+\mu_{i}\right] r_{i j}-\lambda_{j}+\gamma_{i j}=0, \\
\gamma_{i j} \times \alpha_{i j}=0
\end{gathered}
$$

where $\bar{U}_{i}(\cdot)=\log \left(U_{i}(\cdot)\right)$. From (27), we know if carrier $j$ is allocated to UE $i$ (i.e., $\alpha_{i j}>0$ ), the following equation holds.

$$
\left[\bar{U}_{i}^{\prime}\left(r_{i}\right)+\mu_{i}\right] r_{i j}=\lambda_{j}
$$

Otherwise, carrier $j$ is not allocated to UE $i$, we have

$$
\left[\bar{U}_{i}^{\prime}\left(r_{i}\right)+\mu_{i}\right] r_{i j} \leq \lambda_{j}
$$

Note that $\lambda_{j}$ is constant for carrier $j$ and each carrier is assigned to only one UE. Therefore, we can conclude from (28) and (29) that carrier $j$ can be allocated to UE $i$ if the following condition is satisfied.

$$
i^{*}=\arg \max _{i}\left[\bar{U}_{i}^{\prime}\left(r_{i}\right)+\mu_{i}\right] r_{i j} .
$$

It can be observed that the optimal carrier allocation can be carried out with (30) if $\mu_{i}$ is determined for UE $i$. For UEs with inelastic traffic, $\mu_{i}>0$ while $\mu_{i}=0$ for UEs with elastic traffic [17]. Additionally, the marginal utility functions $\bar{U}_{i}^{\prime}\left(r_{i}\right)$ of inelastic UEs are much higher than ones of elastic UEs when the data rate is below $r_{r e q}^{i}$. Thus, for carrier allocation, inelastic UEs could have higher priority than elastic UEs. For UEs of the same traffic type, the priority between UEs depends on $r_{i j}$ affected by the channel gain.

\section{Simulation Results}

We evaluate the performance of the proposed approach via Matlab. Table I shows the initial configuration parameters.

Firstly, we investigate the performance of the proposed approach in terms of system throughput and average utility as depicted in Fig 3. While the proposed method prioritizes inelastic UEs by using different utility function with elastic UEs, it is observed that the average utility of inelastic traffic's UE is higher than the one of elastic UE (two sold lines). To show the gain achievable from using the Wi-Fi, we also consider the case that the Wi-Fi access is not available and PCC and SCCs are allocated only from licensed bands. With the Wi-Fi access, it is shown that the system throughput is significantly improved $(63.0 \%)$ at the high traffic load (bar graph). While elastic traffic is considered more suitable for the Wi-Fi access in the proposed approach, the QoS of elastic

TABLE I

Simulation PARAMETERS

\begin{tabular}{|l|c|}
\hline Parameters & Values \\
\hline \hline ISD & $400 \mathrm{~m}$ \\
\hline Transmit power & $46 \mathrm{~mW}$ \\
\hline System bandwidth & $20 \mathrm{MHz} @ 800 \mathrm{MHz} \& 40 \mathrm{MHz} @ 2 \mathrm{GHz}$ \\
\hline The number of carriers, $N$ & $20(10 @ 800 \mathrm{MHz} \& 10 @ 2 \mathrm{GHz})$ \\
\hline Path Loss model & $119.6+37.2 \log (d)[\mathrm{dB}] @ 800 \mathrm{MHz}$ \\
& $128.1+37.6 \log (d)[\mathrm{dB}] @ 2 \mathrm{GHz}$ \\
\hline WiFi Type & $802.11 \mathrm{~g}(20 \mathrm{MHz} @ 2.4 \mathrm{GHz})$ \\
\hline WiFi AP Tx power & $20 \mathrm{dBm}$ \\
\hline WiFi Path Loss model & $140.7+37.6 \log (d)+21 \log (24 / 2)[\mathrm{dB}]$ \\
\hline WiFi Rate & $\{6,9,12,18,24,36,48,54\}[\mathrm{Mbps}]$ \\
\hline The number of WiFi nodes & 3 \\
\hline The number of UEs, $K$ & varying in $[6,10]$ \\
\hline UE Speed & varying in $\{0,3,10,30,60\}[\mathrm{km} / \mathrm{h}]$ \\
\hline UE traffic types & mixed $($ Inelastic, Elastic) \\
\hline
\end{tabular}




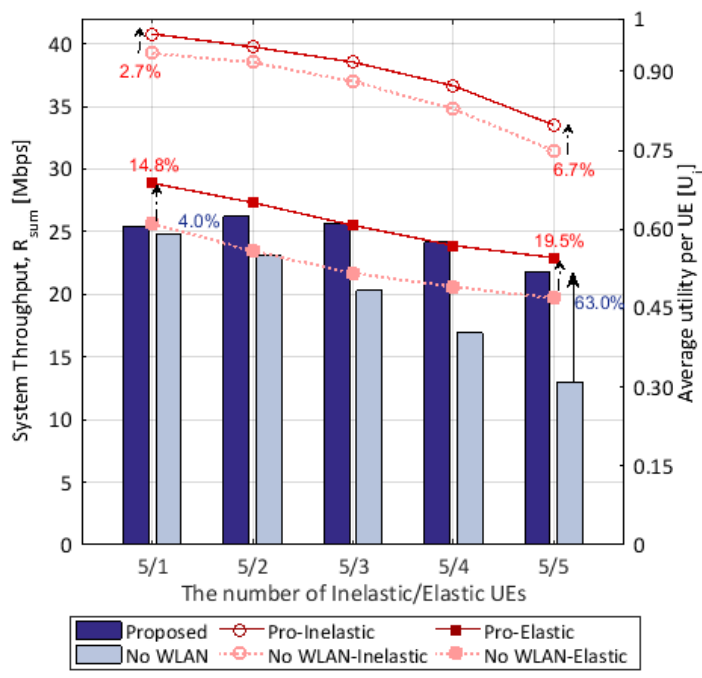

Fig. 3. Improvement of the performance by using the Wi-Fi access

UEs could gain higher than one of inelastic UEs $(19.5 \% \mathrm{vs}$. $6.7 \%$ improvement in average utility) from the use of Wi-Fi.

Fig. 4 shows the performance of the proposed approach considering UEs' mobility. As the reference scheme, we consider the algorithm only considering the Wi-Fi signal strength to allocate UEs to the Wi-Fi link. It is observed that the proposed algorithm gains $39.4 \%$ improvement in the average utility and 52\% improvement in fairness when UEs' move at high speed (average $32 \mathrm{~km} / \mathrm{h}$ ). While the proposed approach estimates and use the UEs' mobility for resource allocation, UEs of high mobility tend to be allocated to licensed bands considering limited coverage of Wi-Fi network. Thus, the proposed approach can allocate better resource to UEs moving with high speed compared to the reference and it leads to improvement in overall QoS and Fairness performance.

\section{CONCLUSiOnS}

In this paper, investigations into resource allocation for heterogeneous networks including WLANs was provided. Ex-

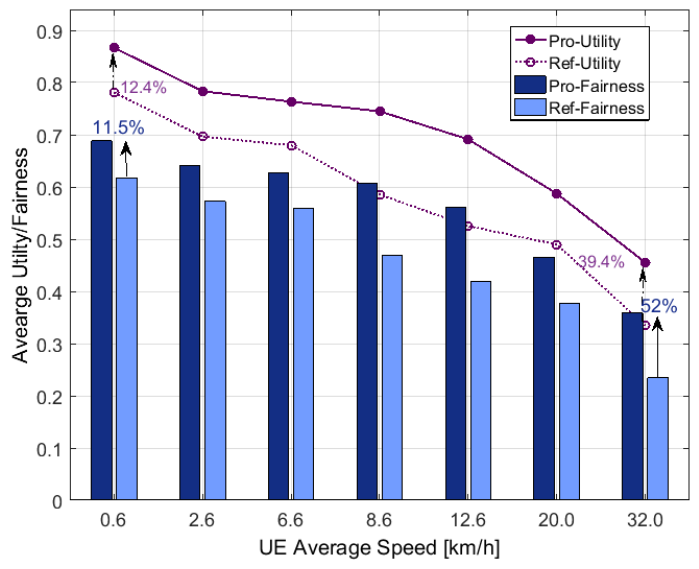

Fig. 4. Performance improvement in terms of average utility and fairness tending the approach of [11], a two-step resource allocation problem was proposed using different utility functions for heterogeneous traffic. The proposed algorithm also considers the different characteristics of the available spectrum bands and traffic as well as the estimated mobility of the users. Simulation results show that the proposed approach is able to support better QoS and spectrum utilization even at high traffic loads and for UEs moving at high speed. Although delay performance in different networks is not considered in this research, there would be non-negligible difference between the delays suffered in heterogeneous networks. In future research, we will consider more comprehensive characteristics of different networks including the delay perspective so that the resource can be allocated more efficiently depending on the characteristics of the heterogeneous networks.

\section{ACKNOWLEDGMENT}

This work is performed as part of SPEED-5G project supported by the EU H2020 under grant agreement no. 671705 .

\section{REFERENCES}

[1] Ericsson, "Mobility report on the pulse of the networked society," White Paper, Nov. 2015.

[2] Nokia Networks, "LTE for Unlicensed Spectrum," White Paper, 2014.

[3] V. Erceg, F. Baboescu, and T. Berham, "Discussion on LTE-WLAN Aggregation (LWA) and LTE WLAN Radio Level Integration with IPsec tunnel (LWIP)," IEEE 802.11-16/0437r1, March. 2016.

[4] Radiosys, "LTE/Wi-Fi Aggregation (LWA)," White Paper, Dec. 2016.

[5] Nokia Networks, "LTE-Advanced Carrier Aggregation Optimization," White Paper, 2014.

[6] M. Katoozian and H. Yanikomeroglu, "Utility-based adaptive radio resource allocation in OFDM wireless networks with traffic prioritization," IEEE Trans. Wireless Commun., vol. 8, no. 1, pp. 66-71, Jan. 2009.

[7] A. Abdelhadi and C. Clancy, "An optimal resource allocation with joint carrier aggregation in 4G-LTE," in Proc. Int'l Conf. Computing, Netw. Commun. (ICNC), California, USA, Feb. 2015, pp. 138-142.

[8] J. Liu, X. Tao, X. Zhou, and Q. Cui, "Utility based resource allocation algorithm with carrier aggregation on unlicensed band," in Proc. Wireless and Optical Commun. Conf. (WOCC), 2015, pp. 180-184.

[9] J. G. Andrews, S. Singh, Q. Ye, X. Lin, and H. S. Dhillon, "An Overview of Load Balancing in HetNets: Old Myths and Open Problems," IEEE Wireless Commun., vol. 21, no. 2, pp. 18-25, April 2014.

[10] S. Goyal, T. B. Le, A. Chincholi, T. Elkourdi, and A. Demir, "On the packet allocation of multi-band aggregation wireless networks," Wireless Netw., Mar. 2017.

[11] H. Lee, S. Vahid, and K. Moessner, "Traffic-aware carrier allocation with aggregation for load balancing," in Proc. European Conf. Netw. Commun. (EuCNC) 2017, 2017.

[12] 3GPP, "Evolved Universal Terrestrial Radio Access (E-UTRA) and Evolved Universal Terrestrial Radio Access Network (E-UTRAN); Overall description; Stage 2," TS 36.300, V14.4.0, Sep. 2017.

[13] H. Lee, S. Vahid, and K. Moessner, "A Survey of Radio Resource Management for Spectrum Aggregation in LTE-Advanced," IEEE Commun. Surveys Tuts., vol. 16, no. 2, pp. 745-760, Second 2014.

[14] R. Yin, G. Yu, A. Maaref, and G. Y. Li, "A Framework for Co-Channel Interference and Collision Probability Tradeoff in LTE LicensedAssisted Access Networks," IEEE Trans. Wireless Commun., vol. 15, no. 9, pp. 6078-6090, Sept. 2016.

[15] G. Bianchi, "Performance analysis of the IEEE 802.11 distributed coordination function," IEEE J. Sel. Areas Commun., vol. 18, no. 3, pp. 535-547, March 2000.

[16] Z. Yang, Q. Yang, F. Fu, and K. S. Kwak, "A novel load balancing scheme in LTE and WiFi coexisted network for OFDMA system," in Proc. Int'l Conf. Wireless Comms. Signal Processing (WCSP), Oct 2013.

[17] N. Chen, P. Hu, X. Tao, and Q. Cui, "Utility Based Energy-Efficient Resource Allocation Algorithm in OFDM System," in Proc. of 2014 IEEE Vehicular Tech. Conf. (VTC2014-Fall), Vancouver, Canada, 2014. 